\title{
Set-Valued Uncertain Process: Definition and Some Properties
}

\author{
Alireza Ghaffari-Hadigheh
}

Received: date / Accepted: date

\begin{abstract}
Some phenomena are developing over time, while they are uncertain sets at each moment. From an uncertain set, we mean an unsharp concept, such as "illness" and "recovery", that is not exactly clear, even for an expert. The values of a parameter that are considered "recovery" would guide one to explain the underlying concept quantitatively. For instance, in recovering from some disease, different levels of health might be assumed. Particularly, at each specific time moment, being healthy to some degree would be measured by belonging parameter values to a set of numbers with a specific belief degree. This set might be extracted using imprecisely observed data, while an expert opinion completely expresses the belief degree. Such concepts would direct one to employ uncertainty theory as a strong axiomatic mathematical framework for modeling human reasoning. Another important feature of these sets is their variation over time. For instance, the set defining "recovery" at the beginning stage of recovery in a disease would be completely or partially different from that at other stages. These characteristics result in considering a sequence of evolving sets over time. Analyzing the behavior of such a sequence motivated us to define the set-valued uncertain process. This concept is a combination of uncertain set, uncertain process, and uncertain sequence. Here, we introduce the main concept. Some properties are extracted and clarified, along with some illustrative examples.
\end{abstract}

Keywords Uncertainty Theory · Uncertain set · Uncertain process · Set-valued uncertain process.

\section{Introduction}

Uncertainty theory [6] initiated in 2007, and later completed in 2009 [9], is an axiomatic framework for modeling human reasoning in decision-making processes. Several concepts exist in this theory, such as uncertain sequence, uncertain set, and uncertain process. An uncertain sequence [6] consists of infinite uncertain variables indexed by integers, mostly used when a variable evolves over another growing element, e.g., time.

Alireza Ghaffari-Hadigheh

Department of Applied Mathematics, Azarbaijan Shahid Madani University, Tabriz-Iran. E-mail: hadigheha@azaruniv.ac.ir 
The concept of convergence is essential for such phenomena and is defined in different ways in the literature. Some of these convergence notions dominate the other, meaning that convergence in one mode implies the other. However, they are not equivalent in general.

Uncertain set [10] is another concept in the literature that is a set-valued function on an uncertainty space and aims to model "unsharp concepts" that are essentially set, though their boundaries are not clearly described. Two common examples are "illness" and "healing". Accurate identifying an uncertain set and quantitatively deriving its parameters are the subject of uncertain set theory. These results would be later applied for more analysis and making practical decisions.

Uncertain process was also introduced by Liu [7] for modeling the evolution of uncertain phenomena over time. For example, the exchange rate of two currencies could be considered as an uncertain process when the environment suggests using the uncertainty theory. The underlying phenomenon would be a single uncertain variable that evolves as time goes forward. Concepts of sample path, uncertainty distribution, independent increment process, extreme value, first hitting time, time integral, and stationary increment process have been introduced for an uncertain process.

Recall that evolving factors in a phenomenon would be a collection of uncertain variables arrayed in a vector or a matrix. In such a situation, the existing results and notions in the uncertain process literature can be generalized to a higher dimension with some possible complexities. For example, the absolute value must be replaced by a suitable norm, and other concepts such as extreme value and first hitting time must be redefined likewise.

Generalization of the concepts may need more consideration when the evolving object over time is an uncertain set. For example, "deteriorating" or "recovery" in a disease are observed over time, while they are not defined sharply and would be expressed as evolving uncertain sets. Let us explain this problem with an example. Consider the disease "dementia", according to WHO [24], it is a chronic symptom and commonly has progressive nature. Cognitive capability declines away from what might be anticipated from typical aging. It affects memory, reasoning, orientation, judgment, calculation and learning capacities, communication, and analysis. However, consciousness is not affected, and the impairment in cognitive function is commonly accompanied and occasionally preceded by a reduction in emotional control, social behavior, or motivation.

It is observed that dementia affects each person differently, depending upon the impact of disease and person's personality before becoming ill. Signs and symptoms categorize the severity of dementia into three stages. The early stage is recognized with signs such as forgetfulness, losing track of time, becoming lost in familiar places. As dementia progresses to the middle stage, the signs and symptoms become more clear and more restricting. For example, some recent events and people's names are forgotten. The most visible symptoms in this stage are getting lost at home, having increasing difficulty with communication, and needing help with personal care. In the severe stage, the patient reveals near-total dependence and inactivity. Memory disturbances are serious, and physical signs become more obvious. For instance, they become more unaware of the time and area, have difficulty remembering relatives and friends, and increasing demand for supported self-care. Moreover, difficulty walking and encountering behavior variations that may increase and be combined with aggression are other remarkable indicators. 
Several medical tests exist to diagnose the development of dementia. For instance, Montreal Cognitive Assessment (MoCA) Test initiated in 2005 [20] and then retrieved in 2017 [21] is one the commonly used tests. It is a one-page 30-point test administered in approximately 10 minutes. The short-term memory recall task; visuospatial abilities, multiple aspects of executive functions; attention, concentration, and working memory; language, and abstract reasoning are assessed during this test. Its scores range between 0 and 30. A score of 26 or over is considered to be normal. Patients without cognitive impairment scored an average of 27.4, while those with mild cognitive impairment scored an average of 22.1, and people with Alzheimer's disease scored an average of 16.2. As mentioned above, the score is compared with some criteria, while a range of scores is used to diagnose the disease severity. Importantly, the boundaries of these ranges are defined by experts. Moreover, the scoring process is carried out by an expert while it is completely dependent on her expertise and reasoning. Further, these sets would not be naturally identical for patients of different ages, suggesting that they evolve over time. Developing a logical methodology would be of interest to find an appropriate pattern of advancing these sets. We refer to this notion as the set-valued uncertain process.

Set-valued processes have been studied using probability theory (See, e.g., [3,22]) and are formally referred to as set-valued stochastic processes. It has a rich theoretical background and a vast range of applications. Especially, it is used in set-valued differential equations along with a Brownian motion (See, e.g., [28]) as well as addressing other problems in fanatical mathematics (See, e.g., [2,27]). The main assumption in these studies is that the probability space is known. These models well work when frequency governs the experiments and enough reliable data exist. However, many phenomena do not obey stochastic rules, and provided data are mostly extracted from experts' opinions. Using probability theory in these situations might be misleading and produce unrealistic results.

One may be tempted to use Fuzzy theory when data are extracted from an expert. The fuzzy process has been studied from different aspects (see for some recent applications in $[26,19])$. Observe that a fuzzy process is a function from $T \times(\Theta, \mathcal{P}, \mathcal{C} r)$ to the set of real numbers, where $(\Theta, \mathcal{P}, \mathcal{C} r)$ is a credibility space [8]. In fact, the object that evolves over a credibility space is a real value, not a fuzzy set. Fuzzy set theory has also been considered in combination with the set-valued stochastic process. Li et al. [4] considered fuzzy set-valued Gaussian processes and Brownian motions, in which the classical Gaussian stochastic process was extended to a case where the process elements are allowed to take values of fuzzy sets, and a new fuzzy Brownian motion was first introduced. Further extensions have been proposed later (See. e.g., [5, 23]). While fuzzy set theory works well in some situations, however, there are some contradictions in the theory that hinders being used everywhere (See [18] for a counter-intuitive example).

This paper introduces the set-valued uncertain process for analyzing an unsharp concept that evolves over time. We consider uncertainty theory as an underlying mathematical structure in our study [18]. The inverse set-valued uncertain process is defined and explained by an example. Membership function and convergence notion are defined. Some illustrative examples are provided for clarifying the results. Findings would suggest a pattern for developing such sets and promoting the decision-making process, say diagnostics in chronic diseases. Collecting data from several patients and implementing an expert's opinion would help to describe the findings quantitatively.

The paper is organized in the following manner. Sec. 2 reviews some necessary facts from the uncertainty theory. In Sec. 3, the concept of set-valued uncertain process is 
introduced. It follows by the definition of a set-valued sample path and presenting some of its properties. These concepts are illustrated using some simple examples. The membership function of a set-valued uncertain process is defined in Sec. 4. We also introduced inverse set-valued uncertain process and belief of observing for an inexact observed quantity. The expected value of a set-valued uncertain process and computational method for its calculation are presented in Sec. 5. Phase distance in a set-valued uncertain process is introduced in Sec. 6 and then used in defining different notions of convergence in Sec. 7. The final section includes a summary of results and sketches further research directions.

\section{Preliminary definitions}

Consider a universal set $\Gamma$, with $\sigma$-algebra $\mathcal{L}$ and uncertain measure $\mathcal{M}$. Each $\Lambda \in \mathcal{L}$ is referred to as an event, and $(\Gamma, \mathcal{L}, \mathcal{M})$ is called an uncertainty space where $\mathcal{M}$ satisfies the following four axioms $[6,9]$.

Axiom 1. (Normality Axiom) $\mathcal{M}\{\Gamma\}=1$ for the universal set $\Gamma$.

Axiom 2. (Duality Axiom) $\mathcal{M}\{\Lambda\}+\mathcal{M}\left\{\Lambda^{c}\right\}=1$ for any event $\Lambda$.

Axiom 3. (Subadditivity Axiom) For every countable sequence of events $\Lambda_{1}, \Lambda_{2}, \ldots$, we have

$$
\mathcal{M}\left\{\bigcup_{i=1}^{\infty} \Lambda_{i}\right\} \leq \sum_{i=1}^{\infty} \mathcal{M}\left\{\Lambda_{i}\right\}
$$

Axiom 4. (Product Axiom) Let $\left(\Gamma_{k}, \mathcal{L}_{k}, \mathcal{M}_{k}\right)$ be uncertainty spaces for $k=1,2, \cdots$ The product uncertain measure $\mathcal{M}$ is an uncertain measure satisfying

$$
\mathcal{M}\left\{\prod_{k=1}^{\infty} \Lambda_{k}\right\}=\bigwedge_{k=1}^{\infty} \mathcal{M}_{k}\left\{\Lambda_{k}\right\}
$$

where $\Lambda_{k}$ are arbitrarily chosen events from $\mathcal{L}_{k}$ for $k=1,2, \cdots$, respectively.

An uncertain variable is a function $\xi$ from an uncertainty space $(\Gamma, \mathcal{L}, \mathcal{M})$ to the set of real numbers such that $\{\gamma \in \Gamma \mid \xi(\gamma) \in B\}$ is an event for any Borel set $B$ of real numbers [6]. An uncertain set is a function $\xi$ from an uncertainty space $(\Gamma, \mathcal{L}, \mathcal{M})$ to a collection of sets of real numbers such that both $\{\gamma \in \Gamma \mid B \subset \xi(\gamma)\}$ and $\{\gamma \in \Gamma \mid \xi(\gamma) \subset$ $B\}$ are events for any Borel set $B$ of real numbers [10]. It has a membership function $\mu(x)$ if for any Borel set $B$ of real numbers, we have

$$
\begin{aligned}
& \mathcal{M}\{\gamma \mid B \subset \xi(\gamma)\}=\inf _{x \in B} \mu(x), \\
& \mathcal{M}\{\gamma \mid \xi(\gamma) \subset B\}=1-\sup _{x \in B^{c}} \mu(x) .
\end{aligned}
$$

Two uncertain sets $\xi_{1}$ and $\xi_{2}$ are independent [14] if for any Borel sets $B_{1}$ and $B_{2}$ of real numbers, we have

$$
\mathcal{M}\left\{\left(\xi_{1}^{*} \subset B_{1}\right) \cap\left(\xi_{2}^{*} \subset B_{2}\right)\right\}=\mathcal{M}\left\{\xi_{1}^{*} \subset B_{1}\right\} \wedge \mathcal{M}\left\{\xi_{2}^{*} \subset B_{2}\right\}
$$

and

$$
\mathcal{M}\left\{\left(\xi_{1}^{*} \subset B_{1}\right) \cup\left(\xi_{2}^{*} \subset B_{2}\right)\right\}=\mathcal{M}\left\{\xi_{1}^{*} \subset B_{1}\right\} \vee \mathcal{M}\left\{\xi_{2}^{*} \subset B_{2}\right\}
$$


where $\xi_{i}^{*}$ are arbitrarily chosen from $\left\{\xi_{i}, \xi_{i}^{c}\right\}, i=1,2$.

An uncertain set $\xi$ is called triangular if it has a membership function

$$
\mu(x)=\left\{\begin{array}{l}
\frac{x-a}{b-a}, \text { if } a \leq x \leq b \\
\frac{x-c}{b-c}, \text { if } b \leq x \leq c
\end{array}\right.
$$

denoted by $(a, b, c)$ where $a, b, c$ are real numbers with $a<b<c$.

\section{Set-valued uncertain process}

Here, we define the set-valued uncertain process that is an uncertain set at each time. Let us define the set-valued uncertain process formally.

Definition 1 Let $(\Gamma, \mathcal{L}, \mathcal{M})$ be an uncertainty space and $T$ be a totally ordered set (hereafter, we call it time). A set-valued uncertain process is a function $\xi_{t}(\gamma)$ from $T \times(\Gamma, \mathcal{L}, \mathcal{M})$ to a collection of sets of real numbers such that both $\left\{\gamma \mid B \subset \xi_{t}(\gamma)\right\}$ and $\left\{\gamma \mid \xi_{t}(\gamma) \subset B\right\}$ are events for any Borel set $B$ of real numbers at any time $t \in T$.

Example 1 Take an uncertainty space $(\Gamma, \mathcal{L}, \mathcal{M})$ to be $\left\{\gamma_{1}, \gamma_{2}, \gamma_{3}\right\}$ with power set and $\mathcal{M}\left\{\gamma_{1}\right\}=0.6, \mathcal{M}\left\{\gamma_{2}\right\}=0.3, \mathcal{M}\left\{\gamma_{3}\right\}=0.2$. It is easy to verify that $\xi_{t}$ defined by

$$
\xi_{t}(\gamma)= \begin{cases}{[t, 3+t],} & \text { if } \gamma=\gamma_{1} \\ {[2 t-1,4+t],} & \text { if } \gamma=\gamma_{2} \\ {[3 t, 5+2 t],} & \text { if } \gamma=\gamma_{3}\end{cases}
$$

is an uncertain set for every time $t \geq 0$.

Definition 2 (Set-valued sample path) Let $\xi_{t}$ be a set-valued uncertain process. For each $\gamma \in \Gamma$, the set-valued function $\xi_{t}(\gamma)$ is called a set-valued sample path of $\xi_{t}$ and its graph is denoted by

$$
\mathcal{G}(\gamma)=\left\{(t, x) \in T \times \mathbb{R}, x \in \xi_{t}(\gamma)\right\}
$$

Definition 3 (Graph of a set-valued uncertain process) For any time $t \in T$ and any event $\Lambda \in \mathcal{L}$, we write $\xi_{t}(\Lambda)=\bigcup_{\gamma \in \Lambda} \mathcal{G}(\gamma)$. The graph of a set-valued uncertain process $\xi_{t}$ is the set

$$
\mathcal{G}\left(\xi_{t}\right)=\bigcup_{\gamma \in \Gamma} \mathcal{G}(\gamma)=\bigcup_{\gamma \in \Gamma}\left\{(t, x) \in T \times \mathbb{R}, x \in \xi_{t}(\gamma)\right\}
$$

A set-valued uncertain process is convex, or closed, or polyhedral if its graph is likewise for every $\gamma \in \Gamma$.

Definition 4 (Lower semi-continuity property) A set-valued sample path $\xi_{t}(\gamma)$ is Lower Semi-Continuous (LSC) at a point $(\tilde{t}, x)$ in its graph if for all neighbourhood of $x$ (open set $U \subset \mathbb{R}$ containing $x$ ), $\left\{t \mid \xi_{t}(\gamma) \cap U \neq \varnothing\right\}$ is an open set in $\mathbb{R}$ containing $\tilde{t}$. In other words, for any sequence of points $\left(t_{n}\right)$ approaching $\tilde{t}$ there is a sequence of points $x_{n} \in \xi_{t_{n}}(\gamma)$ approaching $x$. If, for a specific $\tilde{t} \in T$, this property holds for all points $x \in \xi_{\tilde{t}}$, we say $\xi_{t}(\gamma)$ is LSC at $\tilde{t}$. A set-valued sample path $\xi_{t}(\gamma)$ is LSC if it is LSC for all $t \in T$. A set-valued uncertain process is LSC if all its set-valued sample paths are LSC. 


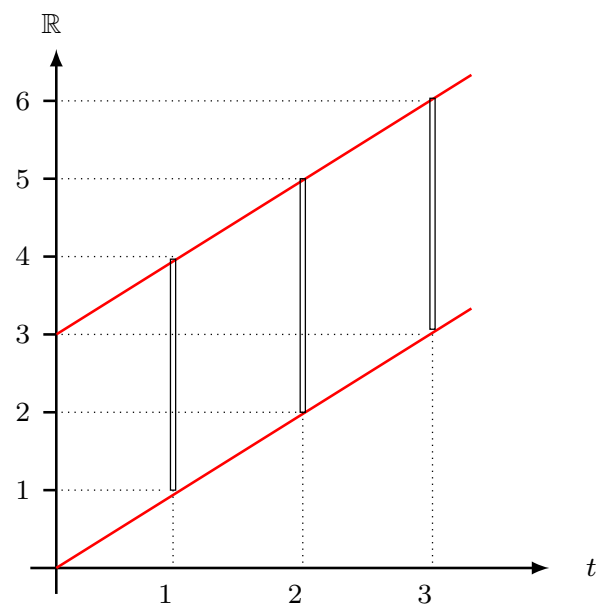

Fig. 1: A set-valued sample path of the set-valued uncertain process in Example 1 for $\gamma=\gamma_{1}$.

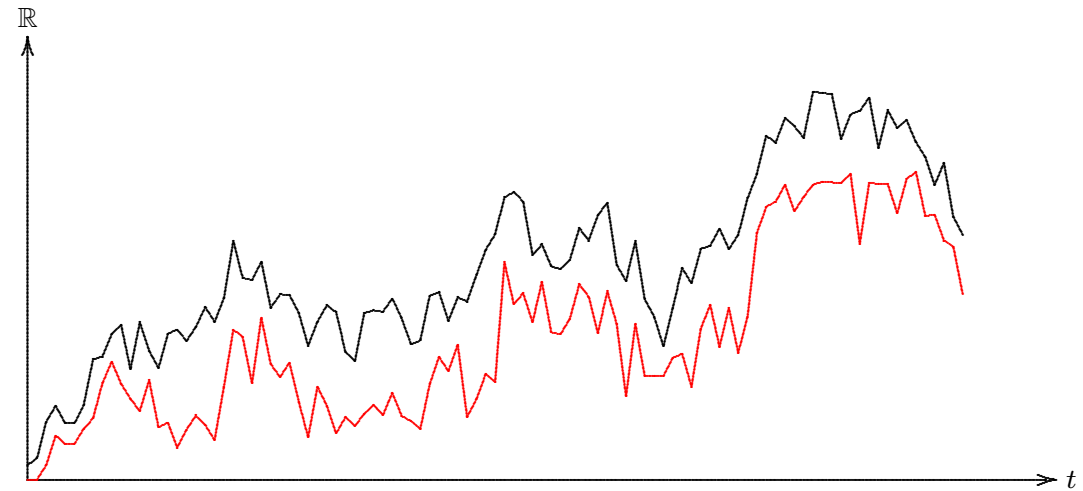

Fig. 2: A sample set-valued path of a set-valued uncertain process.

Remark 1 Fig. 1 depicts the set-valued sample path in Example 1 for $\gamma=\gamma_{1}$ (the region between two red lines). Fig. 2 denotes a typical set-valued sample path; the region between red and black borders. Note that each set-valued sample path is a setvalued function of time $t$. In addition, a set-valued uncertain process can be regarded as a function from an uncertainty space to a collection of set-valued sample paths. A set-valued uncertain process is nonempty if for all $\gamma$ and $t \in T$, we have $\xi_{t}(\gamma) \neq \varnothing$. Furthermore, a set-valued sample path is convex, or closed, or polyhedral if its graph is likewise. Observe that all set-valued sample paths in Example 1 are convex, closed, and polyhedral (See Fig. 1 for $\gamma=\gamma_{1}$ and Fig. 4 a for all $\gamma$ s). The set-valued sample path depicted in Fig. 2 is only closed when it includes the borders, but it is neither convex nor polyhedral. However, both set-valued sample paths in Fig.s 1 and 2 are LSC. 


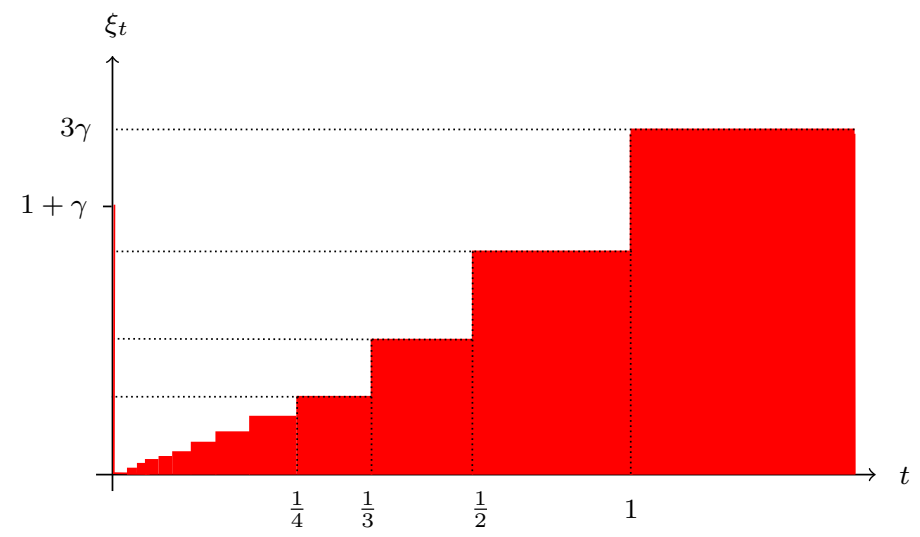

Fig. 3: A typical set-valued sample path which is not LSC.

Remark 2 Observe that all set-valued uncertain processes are not LSC. For example, Let $\Gamma$ be the interval $[0,1]$, with Borel algebra and Lebesgue measure. Define

$$
\xi_{t}(\gamma)= \begin{cases}{[0,3 \gamma]} & \text { if } t \geq 1 \\ {\left[0, \frac{\gamma}{k}\right]} & \text { if } \frac{1}{k+1} \leq t<\frac{1}{k}, \quad(k=1,2,3, \ldots) \\ {[0,1+\gamma]} & \text { if } t=0 .\end{cases}
$$

For $\gamma \geq \frac{1}{2}$, a typical set-valued sample path (red region) is denoted in Fig. 3. It is not hard to verify that for any $\gamma \in(0,1)$, all set-valued sample paths are not LSC at $t=0$ and $x \in(0,1+\gamma] \subset \xi_{0}(\gamma)$.

\section{Membership function}

Definition 5 A set-valued uncertain process $\xi_{t}$ is said to have a membership function $\mu_{t}(x)$ for $t \in T$ if for any Borel set $B$ of real numbers, we have

$$
\mathcal{M}\left\{\gamma \mid B \subset \xi_{t}(\gamma)\right\}=\inf _{x \in B} \mu_{t}(x), \quad \mathcal{M}\left\{\gamma \mid \xi_{t}(\gamma) \subset B\right\}=1-\sup _{x \in B^{c}} \mu_{t}(x)
$$

Theorem 1 Let $\xi_{t}$ be a set-valued uncertain process whose membership function $\mu_{t}(x)$ exists. Then

$$
\mu_{t}(x)=\mathcal{M}\left\{\gamma \mid x \in \xi_{t}(\gamma)\right\}
$$

for any number $x \in \mathbb{R}$, and $t \in T$.

Proof For any number $x \in \mathbb{R}$ and $t \in T$, it follows from (10) that

$$
\mathcal{M}\left\{\gamma \mid x \in \xi_{t}(\gamma)\right\}=\mathcal{M}\left\{\gamma \mid\{x\} \subset \xi_{t}(\gamma)\right\}=\inf _{y \in\{x\}} \mu_{t}(y)=\mu_{t}(x)
$$

The statement is proved. 


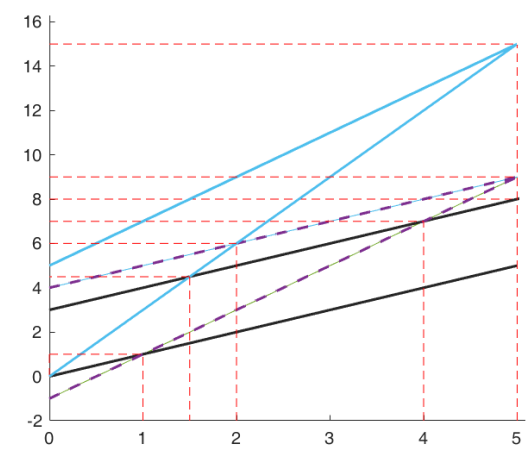

(a) Domain partition

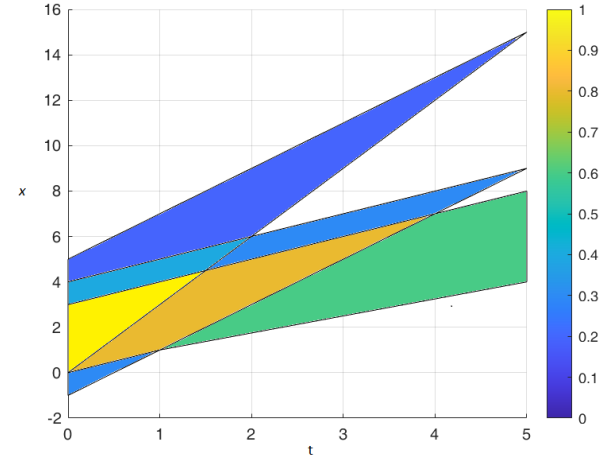

(b) membership function in different colors

Fig. 4: Domain and membership function of the set-valued uncertain process defined by $(8)$.

Example 2 Consider the set-valued uncertain process in Example 1. Different lines partition the domain of its membership function (See Fig. 4a), while is is constant over each part. Table 1 denotes these regions and the membership function values over them. Observe that this membership function is not continuous. Fig. 4b pictures this function by different colors.

Example 3 Consider the uncertainty space $(\Gamma, \mathcal{L}, \mathcal{M})$ be $[0,1]$ with Borel algebra and Lebesgue measure. Define the set-valued uncertain process as

$$
\xi_{t}(\gamma)=\left[t-\frac{\gamma}{1+t}, t+\frac{\gamma}{1+t}\right], \quad \forall \gamma \in[0,1]
$$

for all $t \geq 0$. Note that as $t$ increases, the uncertainty set gets shrunk, meaning that by proceeding in time, the uncertainty vanishes and the uncertain set reduces to the crisp value $t$. We will discuss the convergence of a set-valued uncertain process later in detail. One can verify that for every $t \geq 0$, membership function of (12) is

$$
\mu_{t}(x)=\left\{\begin{array}{cc}
1-(1+t)|x-t|, \text { if } & t-\frac{1}{1+t} \leq x \leq t+\frac{1}{1+t} \\
0, & \text { Otherwise }
\end{array}\right.
$$

Fig.s $5 \mathrm{a}$ and $5 \mathrm{~b}$ denote the uncertain membership function (13) for $0 \leq t \leq 2$ in $3 \mathrm{D}$ and $2 \mathrm{D}$, respectively.

Definition 6 The inverse of a set-valued uncertain process $\xi_{t}$ is defined as $\xi_{t}^{-1}: \mathbb{R} \rightarrow$ $(\Gamma, \mathcal{L}, \mathcal{M})$ by the relationship

$$
\gamma \in \xi_{t}^{-1}(x) \Longleftrightarrow x \in \xi_{t}(\gamma),
$$

for $x \in \mathbb{R}$ and $t \in T$.

Observe that the inverse set-valued uncertain process defines a subset of the universal set $\Gamma$ for each real value $x$. 
Table 1: Domain of the membership function ans its representation on each region in Example 1.

\begin{tabular}{|c|c|c|c|}
\hline$t$ & $x$ & event $\Lambda$ & $\mathcal{M}\{\Lambda\}$ \\
\hline \multirow{5}{*}{$0 \leq t<1$} & $-1+2 t \leq x \leq t$ & $\left\{\gamma_{2}\right\}$ & 0.3 \\
\hline & $t \leq x \leq 3 t$ & $\left\{\gamma_{1}, \gamma_{2}\right\}$ & 0.8 \\
\hline & $3 t \leq x \leq 3+t$ & $\left\{\gamma_{1}, \gamma_{2}, \gamma_{3}\right\}$ & 1 \\
\hline & $3+t \leq x \leq 4+t$ & $\left\{\gamma_{2}, \gamma_{3}\right\}$ & 0.4 \\
\hline & $4+t \leq x \leq 5+2 t$ & $\left\{\gamma_{3}\right\}$ & 0.2 \\
\hline \multirow{5}{*}{$1 \leq t<\frac{3}{2}$} & $t \leq x \leq-1+2 t$ & $\overline{\left\{\gamma_{1}\right\}}$ & 0.6 \\
\hline & $-1+2 t \leq x \leq 3 t$ & $\left\{\gamma_{1}, \gamma_{2}\right\}$ & 0.8 \\
\hline & $3 t \leq x \leq 3+t$ & $\left\{\gamma_{1}, \gamma_{2}, \gamma_{3}\right\}$ & 1 \\
\hline & $3+t \leq x \leq 4+t$ & $\left\{\gamma_{2}, \gamma_{3}\right\}$ & 0.4 \\
\hline & $4+t \leq x \leq 5+2 t$ & $\left\{\gamma_{3}\right\}$ & 0.2 \\
\hline \multirow{5}{*}{$\frac{3}{2} \leq t<2$} & $t \leq x \leq-1+2 t$ & $\left\{\gamma_{1}\right\}$ & 0.6 \\
\hline & $-1+2 t \leq x \leq 3+t$ & $\left\{\gamma_{1}, \gamma_{2}\right\}$ & 0.8 \\
\hline & $3+t \leq x \leq 3 t$ & $\left\{\gamma_{2}\right\}$ & 0.3 \\
\hline & $3 t \leq x \leq 4+t$ & $\left\{\gamma_{2}, \gamma_{3}\right\}$ & 0.4 \\
\hline & $4+t \leq x \leq 5+2 t$ & $\left\{\gamma_{3}\right\}$ & 0.2 \\
\hline \multirow{5}{*}{$2 \leq t<4$} & $t \leq x \leq-1+2 t$ & $\left\{\gamma_{1}\right\}$ & 0.6 \\
\hline & $-1+2 t \leq x \leq 3+t$ & $\left\{\gamma_{1}, \gamma_{2}\right\}$ & 0.8 \\
\hline & $3+t \leq x \leq 4+t$ & $\left\{\gamma_{2}\right\}$ & 0.3 \\
\hline & $4+t \leq x \leq 3 t$ & $\{\varnothing\}$ & 0 \\
\hline & $3 t \leq x \leq 5+2 t$ & $\left\{\gamma_{3}\right\}$ & 0.2 \\
\hline \multirow{5}{*}{$4 \leq t<5$} & $t \leq x \leq 3+t$ & $\left\{\gamma_{1}\right\}$ & 0.6 \\
\hline & $3+t \leq x \leq-1+2 t$ & $\{\varnothing\}$ & 0 \\
\hline & $-1+2 t \leq x \leq 4+t$ & $\left\{\gamma_{2}\right\}$ & 0.3 \\
\hline & $4+t \leq x \leq 3 t$ & $\{\varnothing\}$ & 0 \\
\hline & $3 t \leq x \leq 5+2 t$ & $\left\{\gamma_{3}\right\}$ & 0.2 \\
\hline \multirow{5}{*}{$5 \leq t$} & $t \leq x \leq 3+t$ & $\left\{\gamma_{1}\right\}$ & 0.6 \\
\hline & $3+t \leq x \leq 4+t$ & $\{\varnothing\}$ & 0 \\
\hline & $4+t \leq x \leq-1+2 t$ & $\{\varnothing\}$ & 0 \\
\hline & $-1+2 t \leq x \leq 5+2 t$ & $\{\varnothing\}$ & 0 \\
\hline & $5+2 t \leq x \leq 3 t$ & $\{\varnothing\}$ & 0 \\
\hline
\end{tabular}



(a) 3D graph of uncertain process

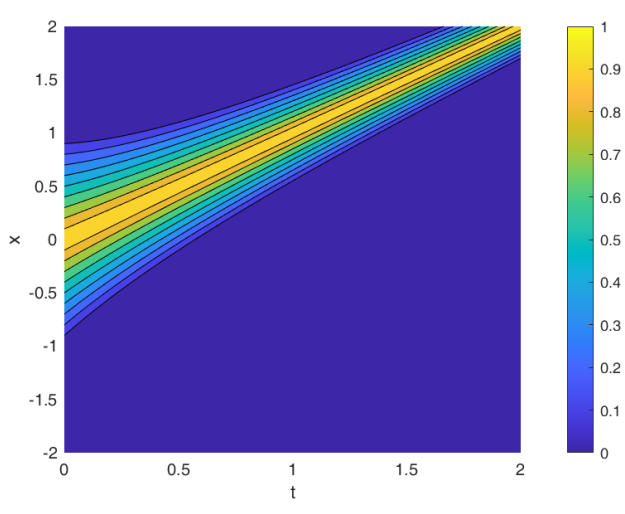

(b) 2D graph of uncertain process

Fig. 5: Graph of the uncertain process defined by (12). 
Example 4 Consider the set-valued uncertain process defined by (12). According to the Definition $6, \gamma \in \xi_{t}^{-1}(x)$ if and only if $x \in\left[t-\frac{\gamma}{1+t}, t+\frac{\gamma}{1+t}\right]$. This means that $\gamma$ must satisfies $(1+t)|x-t| \leq \gamma$, since $\gamma \in[0,1]$. The set of such $\gamma$ 's is empty unless for the given $x, t \geq 0$ satisfies

$$
(1+t)|x-t| \leq 1
$$

Thus, for each real value $x$, we have



Observe that (14) can be simplified for a given $x$. However, finding a closed form for the domain of $t$, and consequently, for $\xi^{-1}(x)$ are out of our interest. Here, we only calculate its value for some specific values of $x$. For instance

$$
\xi_{t}^{-1}(1)=\left\{\begin{array}{ccc}
{[(1+t)|1-t|, 1]} & \text { if } & 0 \leq t \leq \sqrt{2} \\
\varnothing & \text { Otherwise }
\end{array}\right.
$$

and

$$
\xi_{t}^{-1}(4)=\left\{\begin{array}{cll}
{[(1+t)|4-t|, 1]} & \text { if } & \frac{3+\sqrt{21}}{2} \leq t \leq \frac{3+\sqrt{29}}{2} \\
\varnothing & \text { Otherwise. }
\end{array}\right.
$$

Remark 3 Let us elaborate the meaning of inverse set-valued uncertain process $\xi_{t}(\gamma)$ within an example and clarify its deference with the $\xi_{t}^{-1}(x)$. Suppose we are interested in the values that define the unsharp concept of recovery in a disease defined by a set-valued uncertain process, say $\xi_{t}(\gamma)$. Let us fix the time $t$ in the sequel. Here, each value of $x \in \xi_{t}(\gamma)$ denotes a level of recovery that is identified by the membership function $\mu_{t}(x)$. On the other hand, for the fixed observed value $x$ from a patient, we may be interested in knowing which uncertain events would result in observing $x$ as a level of recovery. These events are members of the underlying $\sigma$-algebra over the universal set $\Gamma$ with different belief degrees. As an intuitive case, let $\xi_{t}$ be defined by (8), and for $t=3, x=5.5$ be observed. Then, $\xi_{3}^{-1}(5.5)=\left\{\gamma_{1}, \gamma_{2}\right\}$. This result is valid for all $x \in[5,6]$. Observe that $\mathcal{M}\left\{\gamma_{1}, \gamma_{2}\right\}=0.8$ in this situation. If the set-valued uncertain process is defined by (12), and for $t=3.9$, the observed value is $x=4$, then $\xi_{3.9}(4)=[0.49,1]$ and $\mathcal{M}\left\{\xi_{3.9}(4)\right\}=0.51$.

Definition 7 (Belief of observing) Let $\xi_{t}(\gamma)$ be a set-valued uncertain process defined on the uncertainty space $(\Gamma, \mathcal{L}, \mathcal{M})$, and $\xi_{t}^{-1}(x)$ be its inverse. Belief of observing $x$ as an instance is defined as $\mathcal{M}\left\{\xi_{t}^{-1}(x)\right\}$.

Theorem 2 Let $\xi_{t}(\gamma)$ be a set-valued uncertain process defined on the uncertainty space $(\Gamma, \mathcal{L}, \mathcal{M})$, and $\xi_{t}^{-1}(x)$ be its inverse. Belief of observing $x$ is identical with the membership value of $x$, that is

$$
\mathcal{M}\left\{\xi_{t}^{-1}(x)\right\}=\mu_{t}(x)
$$

Proof Let $x$ be a fixed real number. Then

$$
\mathcal{M}\left\{\xi_{t}^{-1}(x)\right\}=\mathcal{M}\left\{\gamma \mid \gamma \in \xi_{t}^{-1}(x)\right\}=\mathcal{M}\left\{\gamma \mid x \in \xi_{t}(\gamma)\right\}=\mu_{t}(x) .
$$

The proof is complete. 
Definition 8 we say a set-valued uncertain process $\xi_{t}$ is open at a point $(\hat{t}, \hat{x})$ in its graph if, for all neighborhoods $U$ of $\hat{t}$, the point $\hat{x}$ lies in $\operatorname{int}\left(\xi_{\mathrm{U}}\right)$. Equivalently, for any sequence of points $\left(x_{n}\right)$ approaching $\hat{x}$ there is a sequence of points $\left(t_{n}\right)$ approaching $\hat{t}$ such that $x_{n} \in \xi_{t_{n}}$ for all $n$. If, for $x_{0}$ in the range, this property holds for all points $t$ in $\xi^{-1}(\hat{x})$, we say $\xi_{t}$ is open at $\hat{x}$.

Example 5 We show that the set-valued uncertain process, defined by (12) is open at any $(\hat{t}, \hat{x})$ in its graph. This means that $\hat{x} \in \xi_{\hat{t}}(\gamma)$ for all $\gamma \in[0,1]$. Let $\left(x_{n}\right)$ be a sequence of real numbers converging to $\hat{x}$. Thus, $t_{n}$ must satisfies

$$
t_{n}-\frac{\gamma}{1+t_{n}} \leq x_{n} \leq t_{n}+\frac{\gamma}{1+t_{n}} .
$$

Thus, for an appropriate $t_{n}$, we must have

$$
\begin{aligned}
& t_{n}^{2}+\left(1-x_{n}\right) t_{n}-\left(x_{n}+\gamma\right) \leq 0 \\
& t_{n}^{2}+\left(1-x_{n}\right) t_{n}-\left(x_{n}-\gamma\right) \geq 0
\end{aligned}
$$

This means that

$$
x_{n}-\gamma \leq t_{n}^{2}+\left(1-x_{n}\right) t_{n} \leq x_{n}+\gamma .
$$

Without loss of generality, one may assume that

$$
t_{n}^{2}+\left(1-x_{n}\right) t_{n}=\frac{\left(x_{n}-\gamma\right)+\left(x_{n}+\gamma\right)}{2}=x_{n} .
$$

By assuming $t_{n}=x_{n}$, a root of (24) as a sequence of time, we have $\lim _{n \rightarrow \infty} t_{n}=\hat{t}=\hat{x}$. Considering (12), $\hat{x} \in \xi_{\hat{t}=\hat{x}}(\gamma)$, and therefore, this set-valued uncertain process holds openness property.

There is an elegance relation between openness and LSC property of a set-valued uncertain process and its inverse. The following theorem is an adjustment of Proposition 5.4.1 from [1]. The proof is omitted.

Theorem 3 (Openness and LSC property) Any set-valued uncertain process $\xi_{t}(\gamma)$ is $L S C$ at a point $(t, x)$ in its graph if and only if $\xi_{t}^{-1}(x)$ is open at $(x, t)$.

Definition 9 A set-valued uncertain process $\xi_{t}(\gamma)$ defined on the uncertainty space $(\Gamma, \mathcal{L}, \mathcal{M})$ is called totally ordered for $t \in T$ if $\left\{\xi_{t}(\gamma) \mid \gamma \in \Gamma\right\}$ is a totally ordered set. That is, for any given $\gamma_{1}$ and $\gamma_{2} \in \Gamma$, either $\xi_{t}\left(\gamma_{1}\right) \subset \xi_{t}\left(\gamma_{2}\right)$ or $\xi_{t}\left(\gamma_{2}\right) \subset \xi_{t}\left(\gamma_{1}\right)$ holds.

Example 6 Consider Example 3. For a fixed $t$ and $0 \leq \gamma_{1}<\gamma_{2} \leq 1$, we have

$$
t-\frac{\gamma_{2}}{1+t}<t-\frac{\gamma_{1}}{1+t}<t+\frac{\gamma_{1}}{1+t}<t+\frac{\gamma_{2}}{1+t},
$$

that results in $\xi_{t}\left(\gamma_{1}\right) \subset \xi_{t}\left(\gamma_{2}\right)$. Thus, the set-valued uncertain process defined by (12) is totally ordered.

Definition 10 A membership function $\mu_{t}(x)$ is said to be regular for any $t$ if there exists a point $x_{0}(t)$ such that $\mu_{t}\left(x_{0}(t)\right)=1$ and $\mu_{t}(x)$ is unimodal about the mode $x_{0}(t)$. That is, $\mu_{t}(x)$ is increasing on $\left(-\infty, x_{0}(t)\right]$ and decreasing on $\left[x_{0}(t),+\infty\right)$ for any $t \in T$. 
Example 7 Observe that the membership function (13) of the set-valued uncertain process (12) in Example 3 is triangular for all $t \geq 0$. In fact, $\xi_{t}(\gamma)=\left(t-\frac{\gamma}{1+t}, t, t+\frac{\gamma}{1+t}\right)$. Further $\mu_{t}(t)=1$, and therefore the set-valued uncertain process (12) is regular with $x_{0}(t)=t$ for all $t \geq 0$.

Definition 11 Let $\xi_{t}(\gamma)$ be a set-valued uncertain process with membership function $\mu_{t}(x)$. Then the set-valued uncertain process

$$
\mu_{t}^{-1}(\alpha)=\left\{x \in \mathbb{R} \mid \mu_{t}(x) \geq \alpha\right\}, \quad \forall \alpha \in[0,1], t \in T,
$$

is called the inverse membership function of $\xi_{t}(\gamma)$.

Remark 4 Let $\xi_{t}$ be a set-valued uncertain process with inverse membership function $\mu_{t}^{-1}(\alpha)$. Then, for each $t \in T$, the membership function of $\xi_{t}$ is determined by

$$
\mu_{t}(x)=\sup \left\{\alpha \in[0,1] \mid x \in \mu_{t}^{-1}(\alpha)\right\}
$$

Example 8 It easy to verify that the inverse membership function of (13) is

$$
\mu_{t}^{-1}(\alpha)=\left\{x \in \mathbb{R} \mid t-\frac{1-\alpha}{1+t} \leq x \leq t+\frac{1-\alpha}{1+t}\right\}, \alpha \in[0,1], t \geq 0 .
$$

It was proved that a real-valued function $\mu$ is a membership function of an uncertain set if and only if $0 \leq \mu(x) \leq 1$ [12]. Moreover, a function $\mu^{-1}(\alpha)$ is an inverse membership function of an uncertain set if and only if it is a monotone decreasing set-valued function with respect to $\alpha \in[0,1]$ [13]. A combination of these two facts is summarized in the following theorem. The proof is similar to the corresponding proofs in the references mentioned above and omitted.

Theorem 4 (Sufficient and Necessary Condition) A function $\mu_{t}(x)$ is a membership function of a set-valued uncertain process if and only if $0 \leq \mu(x) \leq 1$ for all $t \in T$. Further, a function $\mu_{t}^{-1}(\alpha)$ defined on $(0,1)$ is an inverse membership function of a setvalued uncertain process if and only if it is a monotone decreasing set-valued function with respect to $\alpha \in[0,1]$ for every $t \in T$. That is,

$$
\mu_{t}^{-1}(\alpha) \subset \mu_{t}^{-1}(\beta), \quad \text { if } \alpha>\beta, \forall t \in T .
$$

\section{Expected Value}

The expected value of an uncertain set has been defined in [10]. The expected value for a set-valued uncertain process $\xi_{t}$ for each $t$ is defined similarly, and denoted by $E\left[\xi_{t}\right]$ for each $t \geq 0$. It is important to note that the expected value of the set-valued uncertain process is a real number for each $t \geq 0$ and therefore, expected sample paths for all $t$ define a sample path independent from the uncertainty in the environment but resulted from this situation.

Definition 12 (Expected sample path) Let $\xi_{t}$ be a nonempty set-valued uncertain process, and denote the expected value of $\xi_{t}$ for every $t \geq 0$ by $E\left[\xi_{t}\right]$. The produced sequence $E\left[\xi_{t}\right]$, indexed by $t$ is referred to as expected sample path. 
Recall that if the membership function of an uncertain set is known, one can use the results in [10] to find its expected value, especially when the membership function is regular. We imitate these results and provide formulas for the expected sample path when the membership function $\mu_{t}(x)$ is given.

Theorem 5 Let $\xi_{t}$ be a set-valued uncertain process with membership function $\mu_{t}(x)$ for all real $x$. Then for each $t \in T$, the expected sample path $E\left[\xi_{t}\right]$ is calculated by

$$
E\left[\xi_{t}\right]=x_{0}(t)+\frac{1}{2} \int_{x_{0}(t)}^{+\infty} \sup _{y \geq x} \mu_{t}(y) d x-\frac{1}{2} \int_{-\infty}^{x_{0}(t)} \sup _{y \leq x} \mu_{t}(y) d x
$$

where $x_{0}(t)$ is a point such that $\mu_{t}\left(x_{0}(t)\right)=1$.

Proof The proof is similar to the proof of Theorem $8.22[18]$ and omitted.

Theorem 6 Let $\xi$ be an uncertain set with regular membership function $\mu$. Then

$$
E\left[\xi_{t}\right]=x_{0}(t)+\frac{1}{2} \int_{x_{0}(t)}^{+\infty} \mu_{t}(x) d x-\frac{1}{2} \int_{-\infty}^{x_{0}(t)} \mu_{t}(x) d x
$$

where $x_{0}(t)$ is a point such that $\mu\left(x_{0}(t)\right)=1$.

Proof The proof is similar to the proof of Theorem 8.23 [18] and omitted.

Remark 5 Let us highlight the differences between the set-valued sample path and expected sample path of $\xi_{t}$. The former is a sequence of uncertain sets for each $\gamma$ in the universal set, while the latter is a sequence of real numbers depicting the expected behavior of the process over time independent from what $\gamma$ is. For instance, consider Example 3 and let $\gamma=\frac{1}{2} \in(0,1)$. Then the set-valued sample path is the sequence of sets $\xi_{t}\left(\frac{1}{2}\right)=\left[t-\frac{1}{2(1+t)}, t+\frac{1}{2(1+t)}\right]$ while the expected sample path is $E\left[\xi_{t}\right]=t$.

\section{Phase distance}

The distance of two uncertain sets has been defined in [12]. Here, we adjust this definition for every $t$ in a set-valued uncertain process.

Definition 13 (Difference between two phases) Consider the set-valued uncertain set $\xi_{t}$, and $t_{1}, t_{2} \in T$. The distance between $\xi_{t_{1}}(\gamma)$ and $\xi_{t_{2}}(\gamma)$ for each $\gamma \in \Gamma$ is defined as

$$
d\left(\xi_{t_{1}}(\gamma), \xi_{t_{2}}(\gamma)\right)=E\left[\left|\xi_{t_{1}}(\gamma)-\xi_{t_{2}}(\gamma)\right|\right] .
$$

Obviously, $d\left(\xi_{t_{1}}(\gamma), \xi_{t_{2}}(\gamma)\right)$ is a nonnegative real number. However despite the distance in crisp sets, it is not zero if $t_{1}=t_{2}$ (See Example 10).

In the sequel, we provide some tools for the calculation of the distance between two phases of a set-valued uncertain process. First, we define the Markovian property for a set-valued uncertain process. This property resembles the uncertain renewal process [7] is an uncertain process in which events occur continuously and independently of one another in uncertain times.

Definition 14 ( Markovian property) A set-valued uncertain process has Markovian property if for all $t \geq 0$, and $s>0$ two uncertain sets $\xi_{t}$ and $\xi_{t+s}$ are independent uncertain sets. 
Observe that Markovian property enables one to identify the membership function $\lambda$ of $\xi_{t_{1}}-\xi_{t_{2}}$ or its inverse for the set-valued uncertain process $\xi_{t}$.

The following theorem relates the inverse membership functions of $\mu_{t_{1}}^{-1}$ and $\mu_{t_{2}}^{-1}$ with the inverse membership function of $\xi_{t_{1}}(\gamma)-\xi_{t_{2}}(\gamma)$. The proof is similar to the proof of Theorem 8.18 in [18] and omitted.

Theorem 7 Let $\xi_{t}$ be a set-valued Markovian uncertain process with the inverse membership function $\mu_{t}^{-1}(\alpha)$. Further, let $t_{1}, t_{2} \in T$ be two arbitrary times. Then $\xi_{t_{1}}-\xi_{t_{2}}$ has an inverse membership function,

$$
\lambda^{-1}(\alpha)=\mu_{t_{1}}^{-1}(\alpha)-\mu_{t_{2}}^{-1}(\alpha) .
$$

The following theorem provides a formula for the membership function of $\xi_{t_{1}}-\xi_{t_{2}}$ in terms of the membership function $\mu_{t}(x)$ for the set-valued Markovian uncertain process $\xi_{t}$.

Theorem 8 Let $\xi_{t}$ be a set-valued Markovian uncertain process with the membership function $\mu_{t}(x)$. Then for $0 \leq t_{1} \leq t_{2}$, Let $\xi_{t_{1}}-\xi_{t_{2}}$ has the membership function

$$
\lambda(x)=\sup _{y \in \Re} \mu_{t_{1}}(x+y) \wedge \mu_{t_{2}}(y) .
$$

Proof Let $\lambda(x)$ be the membership function of $\xi_{t_{1}}-\xi_{t_{2}}$. According to Theorem 8.18 in [18], we define $f\left(\xi_{t_{1}}, \xi_{t_{2}}\right)=\xi_{t_{1}}-\xi_{t_{2}}$. Considering the Markovian property of $\xi_{t}$, two uncertain sets $\xi_{t_{1}}$ and $\xi_{t_{2}}$ are independent with inverse membership functions $\mu_{t_{1}}^{-1}$ and $\mu_{t_{2}}^{-1}$ respectively. Thus,

$$
\lambda^{-1}(\alpha)=\mu_{t_{1}}^{-1}(\alpha)-\mu_{t_{2}}^{-1}(\alpha) .
$$

For any given real number $x$, let $\lambda(x)=\beta$. It holds

$$
\lambda^{-1}(\beta)=\mu_{t_{1}}^{-1}(\beta)-\mu_{t_{2}}^{-1}(\beta) .
$$

Since $x \in \lambda^{-1}(\beta)$, there exist real numbers $x_{1} \in \mu_{t_{1}}^{-1}(\beta)$ and $x_{2} \in \mu_{t_{2}}^{-1}(\beta)$ such that $x_{1}-x_{2}=x$. Noting that $\mu_{t_{i}}\left(x_{i}\right) \geq \beta$ for $i=1,2$, we have

$$
\lambda(x)=\beta \leq \mu_{t_{1}}\left(x_{1}\right) \wedge \mu_{t_{2}}\left(x_{2}\right),
$$

and then

$$
\lambda(x) \leq \sup _{x_{1}-x_{2}=x} \mu_{t_{1}}\left(x_{1}\right) \wedge \mu_{t_{2}}\left(x_{2}\right) .
$$

On the other hand, assume $x_{1}$ and $x_{2}$ are two real numbers with $x_{1}-x_{2}=x$. Let

$$
\mu_{t_{1}}\left(x_{1}\right) \wedge \mu_{t_{2}}\left(x_{2}\right)=\beta \text {. }
$$

Again, using Theorem 8.18 in [18], we have

$$
\lambda^{-1}(\beta)=\mu_{t_{1}}^{-1}(\beta)-\mu_{t_{2}}^{-1}(\beta) .
$$

Noting that $x_{i} \in \mu_{t_{i}}^{-1}(\beta)$ for $i=1,2$, we have

$$
x=x_{1}-x_{2} \in \mu_{t_{1} 1}^{-1}(\beta)-\mu_{t_{2}}^{-1}(\beta)=\lambda^{-1}(\beta) .
$$

Hence

and then

$$
\lambda(x) \geq \beta=\mu_{t_{1}}\left(x_{1}\right)-\mu_{t-2}\left(x_{2}\right),
$$

$$
\lambda(x) \geq \sup _{x_{1}-x_{2}=x} \mu_{t_{1}}\left(x_{1}\right) \wedge \mu_{t_{2}}\left(x_{2}\right) .
$$

The statement follows from (33) and (34) with $x_{2}=y$ and $x_{1}=x+y$. 
Observe that this theorem provides a formula for identifying the membership function of $\xi_{t_{1}}-\xi_{t_{2}}$, however, one may find it costly. In this case, one can use (26) when $\lambda^{-1}$ is available.

Example 9 Consider Example 3 and let $0 \leq t_{1} \leq t_{2} \leq 1$. Further, let $\xi_{t}$ be defined by (12) and have Markovian property. Since both $\mu_{t_{1}}$ and $\mu_{t_{2}}$ are triangle membership functions, then $\xi_{t_{1}}-\xi_{t_{2}}=(a, b, c)$ is a triangular uncertain set (See Example 8.25 [18]), where $a=t_{1}-t_{2}-\left(\frac{1}{1+t_{1}}+\frac{1}{1+t_{2}}\right), b=t_{1}-t_{2}$ and $c=t_{1}-t_{2}+\left(\frac{1}{1+t_{1}}+\frac{1}{1+t_{2}}\right)$. In other words, the membership function of $\xi_{t_{1}}-\xi_{t_{2}}$ is

$$
\lambda(x)=\frac{x-\left[\left(t_{1}-t_{2}\right)-\left(\frac{1}{1+t_{1}}+\frac{1}{1+t_{2}}\right)\right]}{\frac{1}{1+t_{1}}+\frac{1}{1+t_{2}}}
$$

when $t_{1}-t_{2}-\left(\frac{1}{1+t_{1}}+\frac{1}{1+t_{2}}\right) \leq x \leq t_{1}-t_{2}$,

$$
\lambda(x)=-\frac{x-\left[\left(t_{1}-t_{2}\right)+\left(\frac{1}{1+t_{1}}+\frac{1}{1+t_{2}}\right)\right]}{\frac{1}{1+t_{1}}+\frac{1}{1+t_{2}}}
$$

when $t_{1}-t_{2} \leq x \leq t_{1}-t_{2}+\left(\frac{1}{1+t_{1}}+\frac{1}{1+t_{2}}\right)$, and $\lambda(x)=0$ for other values of $x$. Moreover, $\lambda$ is regular since $\lambda\left(t_{1}-t_{2}\right)=1$. Observe that for $t_{1}=t_{2}=\hat{t}$, we have

$$
\xi_{\hat{t}}-\xi_{\hat{t}}=\left(-\frac{2}{1+\hat{t}}, 0, \frac{2}{1+\hat{t}}\right)
$$

The following theorem resembles Theorem 8.31 in [17], the proof is omitted.

Theorem 9 Let $\xi_{t}$ be a set-valued Markovian uncertain process. For $0 \leq t_{1}<t_{2}$, the distance between $\xi_{t_{1}}$ and $\xi_{t_{2}}$ is

$$
d\left(\xi_{t_{1}}, \xi_{t_{2}}\right)=\frac{1}{2} \int_{0}^{+\infty}\left(\sup _{|y| \geq x} \lambda(y)+1-\sup _{|y|<x} \lambda(y)\right) d x
$$

where $\lambda$ is the membership function of $\xi_{t_{1}}-\xi_{t_{2}}$.

Example 10 Let us determine the distance between $\xi_{t_{1}}$ and $\xi_{t_{2}}$ for the set-valued uncertain process in Example 3, with the assumption that it holds Markovian property. Recall the membership of $\xi_{t_{1}}-\xi_{t_{2}}$, calculated in Example 9. We only consider the case when $t_{1} \geq t_{2}+1 \geq 0$. Other cases can be processed similarly with some considerations on the boundaries of integrals. With this consideration, $t_{1}-t_{2}-\left(\frac{1}{1+t_{1}}+\frac{1}{1+t_{2}}\right)$ is positive. Therefore, (37) reduces to

$$
\begin{gathered}
d\left(\xi_{t_{1}}, \xi_{t_{2}}\right)=\frac{1}{2}\left[\int_{t_{1}-t_{2}-\left(\frac{1}{1+t_{1}}+\frac{1}{1+t_{2}}\right)}^{t_{1}-t_{2}} \sup _{|y| \geq x} \lambda(y)+1-\sup _{|y|<x} \lambda(y) d x\right. \\
\left.+\int_{t_{1}-t_{2}}^{t_{1}-t_{2}+\left(\frac{1}{1+t_{1}}+\frac{1}{1+t_{2}}\right)} \sup _{|y| \geq x} \lambda(y)+1-\sup _{|y|<x} \lambda(y) d x\right] .
\end{gathered}
$$


Observe that when $t_{1}-t_{2}-\left(\frac{1}{1+t_{1}}+\frac{1}{1+t_{2}}\right) \leq x \leq t_{1}-t_{2}$,

$$
\sup _{|y| \geq x} \lambda(y)=1 \text {, and } \sup _{|y|<x} \lambda(y)=\lambda(x) .
$$

Moreover, when $t_{1}-t_{2} \leq x \leq t_{1}-t_{2}+\left(\frac{1}{1+t_{1}}+\frac{1}{1+t_{2}}\right)$,

$$
\sup _{|y| \geq x} \lambda(y)=\lambda(x), \text { and } \sup _{|y|<x} \lambda(y)=1 .
$$

Thus (38) reduces to

$$
d\left(\xi_{t_{1}}, \xi_{t_{2}}\right)=\frac{1}{2}\left[\int_{t_{1}-t_{2}-\left(\frac{1}{1+t_{1}}+\frac{1}{1+t_{2}}\right)}^{t_{1}-t_{2}} 2-\lambda(x) d x+\int_{t_{1}-t_{2}}^{t_{1}-t_{2}+\left(\frac{1}{1+t_{1}}+\frac{1}{1+t_{2}}\right)} \lambda(x) d x\right]
$$

After some algebraic manipulation, we have

$$
d\left(\xi_{t_{1}}, \xi_{t_{2}}\right)=\frac{t_{1}+t_{2}+2}{\left(t_{1}+1\right)\left(t_{2}+1\right)}
$$

Observe that when $t_{1}=t_{2}=\hat{t}$ we have

$$
d\left(\xi_{\hat{t}}, \xi_{\hat{t}}\right)=\frac{2 \hat{t}+2}{(\hat{t}+1)(\hat{t}+1)}=\frac{2}{(\hat{t}+1)},
$$

which is not zero.

\section{Convergence of a set-valued uncertain process}

Consider a set-valued uncertain process $\xi_{t}$ and let $\xi$ be a fixed uncertain set for a specific $\gamma$. First observe that $d\left(\xi_{t}(\gamma)-\xi(\gamma)\right)$ is an uncertain sequence indexed by time and each element of this sequence is an uncertain variable that depends on $\gamma$. Therefore, different convergence notions can be defined for a set-valued uncertain process.

Definition 15 The set-valued uncertain process $\xi_{t}$ is said to be convergent a.s. to $\xi$ if there exists an event $\Lambda$ with $\mathcal{M}\{\Lambda\}=1$ such that

$$
\lim _{t \rightarrow \infty} d\left(\xi_{t}(\gamma)-\xi(\gamma)\right)=0
$$

for every $\gamma \in \Lambda$.

Example 11 Let the uncertainty space $(\Gamma, \mathcal{L}, \mathcal{M})$ be $[0,1]$ with Borel algebra and Lebesgue measure. Consider the set-valued uncertain process as

$$
\xi(\gamma)=\{t\}, \quad \forall \gamma \in[0,1]
$$

for $t \geq 0$. Observe that (43) is a special uncertain set with the following membership function

$$
\mu_{t}(x)=\left\{\begin{array}{lll}
1 & \text { if } & x=t \\
0 & \text { Otherwise }
\end{array}\right.
$$


Using Theorem 8 , the membership function of $\xi_{t}-\xi$ is

$$
\begin{aligned}
\lambda(x) & =\sup _{y \in \mathbb{R}} \mu_{t}(x+y) \wedge \mu(y) \\
& =\mu_{t}(x+t) \wedge \mu(t)=\mu_{t}(x+t) \wedge 1=\mu_{t}(x+t),
\end{aligned}
$$

where $\mu_{t}(x)$ is calculated as (13). Thus

$$
\lambda(x)=\left\{\begin{array}{cc}
1-(1+t)|x|, \text { if } & -\frac{1}{1+t} \leq x \leq \frac{1}{1+t} \\
0, & \text { Otherwise }
\end{array}\right.
$$

Observe that for every $x \geq 0$ we have

$$
\sup _{|y|<x} \lambda(y)=\sup _{|y|<\min \left\{x, \frac{1}{1+t}\right\}} \lambda(y)=\sup _{|y|<\min \left\{x, \frac{1}{1+t}\right\}} 1-(1+t)|y|=1 .
$$

Moreover, for $0 \leq x \leq \frac{1}{1+t}$ we have

$$
\sup _{|y| \geq x} \lambda(y)=\lambda(x)=1-(1+t) x,
$$

and for $x \geq \frac{1}{1+t}$ it holds

$$
\sup _{|y| \geq x} \lambda(y)=0
$$

Therefore,

$$
d\left(\xi_{t}, \xi\right)=\frac{1}{2} \int_{0}^{\frac{1}{1+t}} 1-(1+t) x d x=\frac{1}{4(1+t)} .
$$

Obviously, when $t \rightarrow \infty, d\left(\xi_{t}, \xi\right)$ vanishes, proving the a.s. convergence of $\xi_{t}$ in Example 3 to the singleton set $\{t\}$ (See Fig. 5).

Sometimes, the limit uncertain set of a set-valued uncertain process in unknown. In this case, we may have the following uniformaly a.s. convergence concept.

Definition 16 The set-valued uncertain process $\xi_{t}$ is said to be uniformaly convergent a.s if there exists an event $\Lambda$ with $\mathcal{M}\{\Lambda\}=1$ such that for any $\varepsilon>0$, there exist a fix $t$ with $t_{1} \geq t$ and $t_{2} \geq t$,

$$
d\left(\xi_{t_{1}}(\gamma)-\xi_{t_{1}}(\gamma)\right)<\varepsilon
$$

for every $\gamma \in \Lambda$.

Example 12 Consider Example 11, and the special case $t_{1} \geq t_{2}+1 \geq 0$. When both of them are enough large, one can easily deduce from (40) and (41) that $d\left(\xi_{t_{1}}(\gamma)-\xi_{t_{1}}(\gamma)\right)$ reduces to zero for enough large $t_{1}$ and $t_{2}$. Thus, the set-valued uncertain process $\xi_{t}$ defined by (12) is uniformaly convergent a.s.

Analogous to uncertain sequences [18], other types of convergence can be defined.

Definition 17 The set-valued uncertain process $\xi_{t}$ is said to be uniformaly a.s. convergent in measure to $\xi$ if

$$
\lim _{t \rightarrow \infty} \mathcal{M}\left\{d\left(\xi_{t}-\xi\right) \geq \varepsilon\right\}=0,
$$

for every $\varepsilon>0$. 
Definition 18 The set-valued uncertain process $\xi_{t}$ is said to be convergent in mean to $\xi$ if

$$
\lim _{t \rightarrow \infty} E\left[d\left(\xi_{t}-\xi\right)\right]=0
$$

Definition 19 Let $\mu_{t}(x)$ and $\mu(x)$ be membership functions of the set-valued uncertain process $\xi_{t}$ and the uncertain set $\xi$, respectively. We say $\xi_{t}$ pointwise converges in membership function to $\xi$ if for all $x \in \mathbb{R}$ and for all $\varepsilon>0$, there exists $\hat{t} \in T$ such that for $t \geq \hat{t}$,

$$
\left|\mu_{t}(x)-\mu(x)\right|<\varepsilon \text {. }
$$

Recall that the convergence in membership function defined by Def. 19 is pointwise. Here, we define uniform convergence of a set-valued uncertain process in membership function.

Definition 20 Let $\mu_{t}(x)$ and $\mu_{t}(x)$ be membership functions of the set-valued uncertain process $\xi_{t}$ and the uncertain set $\xi$, respectively. We say $\xi_{t}$ uniformaly converges in membership function to $\xi$ if for all $\varepsilon>0$, there exists $\hat{t} \in T$ such that for $t \geq \hat{t}$ and for all $x \in \mathbb{R}$

$$
\left|\mu_{t}(x)-\mu(x)\right|<\varepsilon \text {. }
$$

One may define Cauchy criterion for uniform convergence in membership function. We leave details to the interested reader.

Example 13 Consider the membership functions (13) and (44) of the set-valued uncertain process (12) and (43), respectively. One can verify promptly that for any $\varepsilon>0$, and enough large $t$, when $x \notin\left[t-\frac{1}{1+t}, t+\frac{1}{1+t}\right]$ then

$$
\left|\mu_{t}(x)-\mu(x)\right|=|0-0|=0 .
$$

When $x \in\left[t-\frac{1}{1+t}, t+\frac{1}{1+t}\right]$ and $x \neq t$, then $(1+t)|x-t| \leq 1$, or $1-(1+t)|x-t| \geq 0$, and thus

$$
\left|\mu_{t}(x)-\mu(x)\right|=|1-(1+t)| x-t|-0| \geq 0 .
$$

Observe that the left-hand-side of (52) would be larger than the given $\varepsilon$ for some $x \in\left[t-\frac{1}{1+t}, t+\frac{1}{1+t}\right]$. This means that the convergence in membership function in this instance is not uniform.

\section{Concluding remarks}

We introduced the notion of a set-valued uncertain process. Set-valued sample path and its LSC and openness properties were established. The membership function of a setvalued uncertain process was also defined. Inverse membership function was explained, and sufficient and necessary condition of being an inverse membership function was also constructed. The expected sample path was introduced, and its difference with the set-valued sample path was clarified. Additionally, a set-valued Markovian uncertain process was defined that helps us to propose different convergence notions of a setvalued uncertain process.

As a future work direction, we are interested in investigating more concepts in this paradigm, such as independent increment, extreme values theorem, first hitting time, time integral, and stationary increment for the set-valued uncertain process. 


\section{Declarations}

Funding: Not applicable

Conflict of interest: The authors declare that they have no conflicts of interest.

Availability of data and material: Not applicable

Code availability: Not applicable

Ethics approval: the research involves no human participants and animals and consequently no need for informed consent.

Consent to participate: Not applicable

Consent for publication: Not applicable

\section{References}

1. Borwein, Jonathan, and Adrian S. Lewis. Convex analysis and nonlinear optimization: theory and examples. Springer Science \& Business Media, 2010.

2. Feinstein, Zachary, and Birgit Rudloff. A supermartingale relation for multivariate risk measures, Quantitative Finance 18, no. 12 (2018): 1971-1990.

3. Kisielewicz, Michał. Stochastic differential inclusions and applications. New York: Springer, 2013.

4. Li, Shoumei, and Li Guan. Fuzzy set-valued Gaussian processes and Brownian motions. Information Sciences. 177, no. 16 (2007): 3251-3259.

5. Li, Shoumei, and Aihong Ren. Representation theorems, set-valued and fuzzy set-valued Ito integral. Fuzzy Sets and Systems. 158, no. 9 (2007): 949-962.

6. Liu, Baoding, Uncertainty Theory, 2nd edn, Springer-Verlag, Berlin, 2007.

7. Liu, Baoding, Fuzzy process, hybrid process and uncertain process, Journal of Uncertain Systems, Vol.2, No.1, 3-16, 2008.

8. Liu, Baoding. Fuzzy process, hybrid process and uncertain process. Journal of Uncertain systems. 2, no. 1 (2008): 3-16.

9. Liu, Baoding, Some research problems in uncertainty theory, Journal of Uncertain Systems, Vol.3, No.1, 3-10, 2009.

10. Liu, Baoding, Uncertain set theory and uncertain inference rule with application to uncertain control, Journal of Uncertain Systems, Vol.4, No.2, 83-98, 2010.

11. Liu, Baoding, Uncertainty Theory: A Branch of Mathematics for Modeling Human Uncertainty, Springer-Verlag, Berlin, 2010.

12. Liu, Baoding, Uncertain logic for modeling human language, Journal of Uncertain Systems, Vol.5, No.1, 3-20, 2011.

13. Liu, Baoding, Membership functions and operational law of uncertain sets, Fuzzy Optimization and Decision Making, Vol.11, No.4, 387-410, 2012.

14. Liu, Baoding, A new definition of independence of uncertain sets, Fuzzy Optimization and Decision Making, Vol.12, No.4, 451-461, 2013.

15. Liu, Baoding, Extreme value theorems of uncertain process with application to insurance risk model, Soft Computing, Vol.17, No.4, 549-556, 2013.

16. Liu, Baoding, Uncertainty distribution and independence of uncertain processes, Fuzzy Optimization and Decision Making, Vol.13, No.3, 259-271, 2014.

17. Liu, Baoding, Uncertainty Theory, 4th edn, Springer-Verlag, Berlin, 2015.

18. Liu, Baoding, Uncertainty Theory, 5th edn, Springer-Verlag, Berlin, 2020.

19. Luo, Win-Jet, Kuen-Suan Chen, Chun-Min Yu, and Ting-Hsin Hsu. The fuzzy process quality evaluation model for the STB quality characteristic of machining. Applied Sciences. 10, no. $22(2020): 8272$.

20. Nasreddine, Ziad S., Natalie A. Phillips, Valrie Bdirian, Simon Charbonneau, Victor Whitehead, Isabelle Collin, Jeffrey L. Cummings, and Howard Chertkow. The Montreal Cognitive Assessment, MoCA: a brief screening tool for mild cognitive impairment. Journal of the American Geriatrics Society 53, no. 4 (2005): 695-699.

21. "Montreal Cognitive Assessment (MoCA) Test: Scoring \& Accuracy". Verywell. Retrieved 12 June 2017. 
22. Schmelzer, Bernhard. Set-valued stochastic processes and sets of probability measures induced by stochastic differential equations with random set parameters. In Combining Soft Computing and Statistical Methods in Data Analysis, pp. 561-568. Springer, Berlin, Heidelberg, 2010.

23. Wang, Dabuxilatu, and Liang Zhang. A fuzzy set-valued autoregressive moving average model and its applications. Symmetry 10, no. 8 (2018): 324.

24. https://www.who.int/news-room/fact-sheets/detail/dementia

25. Yao K, Inclusion relationship of uncertain sets, Journal of Uncertainty Analysis and Applications, Vol.3, Article 13, 2015.

26. You, Cuilian, and Le Bo. Option pricing based on a type of fuzzy process. Journal of Ambient Intelligence and Humanized Computing (2021): 1-15.

27. Zhang, Junfei, and Shoumei Li. Quanto European Option Pricing With Ambiguous Return Rates and Volatilities. IEEE Transactions on Fuzzy Systems 25, no. 2 (2016): 417-424.

28. Zhu, Jialu, Yong Liang, and Weiyin Fei. On uniqueness and existence of solutions to stochastic set-valued differential equations with fractional Brownian motions, Systems Science 8 Control Engineering 8, no. 1 (2020): 618-627. 